\title{
Exploring the origin of small-x saturation in the collinear approach
}

\author{
A. M. Snigirev $\circledast^{1,2}$ and G. M. Zinovjev ${ }^{3}$ \\ ${ }^{1}$ Skobeltsyn Institute of Nuclear Physics, Lomonosov Moscow State University, 119991 Moscow, Russia \\ ${ }^{2}$ Bogoliubov Laboratory of Theoretical Physics, JINR, 141980 Dubna, Russia \\ ${ }^{3}$ Bogolyubov Institute for Theoretical Physics, National Academy of Sciences of Ukraine, \\ Kiev 03143, Ukraine
}

(Received 4 October 2019; published 11 November 2019)

\begin{abstract}
A modification of the collinear evolution equations as an appropriate approach to improving the behavior of parton distribution functions in the region of small longitudinal momentum fractions and to finding more theoretical arguments to clarify the possible appearance of the saturation regime is suggested. It is argued that parton diffusion in the rapidity space at large parton densities along the space-time evolution could result in the emergence of a natural saturation scale on which freezing actually occurs.
\end{abstract}

DOI: $10.1103 /$ PhysRevD.100.094008

\section{INTRODUCTION}

Nowadays, it is widely recognized the hadron interactions at very high energies are driven by the states with very high densities of partons (quarks and gluons), in particular, with small longitudinal momentum fractions $x$. The routine theoretical framework for analyzing such systems is essentially grounded on the QCD collinear factorization in which the calculated cross sections are decomposed in the perturbative coefficient functions and nonperturbative parton densities of which evolvement is treated according the Dokshitzer-Gribov-Lipatov-Altarelli-Parisi (DGLAP) equations [1-4]. Already, these linear equations qualitatively capture the traits associated with an increase in the gluon densities at small $x$ with extremely large $Q^{2}$ values. The latter turn out also quite instrumental, for example, to justify by neglecting any type of higher-twist corrections and some perturbative resummation contributions. An idea to follow the evolution within the perturbative paradigm and to evaluate the leading contributions at small $x$ for not very large $Q^{2}$ led to the development of the Balitsky-FadinKuraev-Lipatov (BFKL) approach associated to so-called high-energy factorization. However, resolving the corresponding BFKL equations [5-7] exhibits a very strong raise (powerlike) of the gluon density at small $x$ that is stronger than the experimental data analysis demonstrates and leads to an apparent violation of unitarity at very small $x$. It signals some theoretical problems generated by the appearance of an infrared instability related to a diffusion with the

Published by the American Physical Society under the terms of the Creative Commons Attribution 4.0 International license. Further distribution of this work must maintain attribution to the author(s) and the published article's title, journal citation, and DOI. Funded by SCOAP ${ }^{3}$. rapidity evolution and the consistent description of QCD coupling constant $\alpha_{s}$ behavior that should reflect a very sophisticated interplay of perturbative and nonperturbative QCD physics. Apparently, both look like an ensuing result of taking into account the linear evolution only with resummation in these approaches. From the phenomenological point of view, an observation of a scaling law in a wide range of small $x$ and $Q^{2}$ was done [8], thereby demonstrating an onset of the saturation scale. This fact is quite interesting because it may provide a perturbative scale in the high-density region of small $x$ where linear evolution approximation works and provides, in a sense, a boundary condition to the linear evolution equations. In fairness, remember that it was argued a long time ago $[9,10]$ that eventually the system under consideration should enter a new regime, in which the rate of growing gluon density slows down and saturates, thus possibly curing a potential conflict with unitarity of the underlying scattering. Actually, the restoration of the unitarity in the high-energy limit of QCD remains a challenging problem, although several approaches, drawing a scenario with nonlinear behavior, have been explored in past years (see, for example, Refs. [11-18] and references therein), but those allow us to conclude only that we are still essentially no closer to knowing where the problem solution lies besides the very general claim about the nonperturbative finite-density effects that are left out entirely from the BFKL evolution. The interest in physics of the high-density regime of small- $x$ QCD is greatly increasing and dictated by an avalanche of experimental data on collisions of relativistic heavy ions overwhelming this area of research in the last few decades.

Meanwhile, there is another opportunity to address the problem in the framework of well-known DGLAP approach that we would like to draw attention to in this paper. It concerns one possible modification of collinear 
timelike evolution equations that was also discussed a long time ago $[19,20]$ as well as in the context of increasing parton multiplicity in electron-positron annihilation into hadrons. We adapt this modification for the spacelike evolution of parton distribution functions and demonstrate it develops a saturated regime of the color glass condensate [21]. This is a regime of strong color fields in which nonlinear dynamics comes to the perceptible play and signals, thereby, an appearance of the natural saturation scale. The evolution is getting frozen on that scale, in fact, indicating also the universality of both phenomena. Actually, such an approach is treated as an effective theory of highenergy scattering successfully describing the data measured in experiments.

The paper is organized as follows. In Sec. II, we briefly review the principal features of the DGLAP evolution in order to be clear and to introduce the notations. The particular modified QCD evolution is discussed in Sec. III. In Sec. IV, the extension to the double parton distribution functions is considered. The possible phenomenological issues are discussed in Sec. V, together with some conclusions.

\section{COLLINEAR EVOLUTION}

One may take the value of the hard scale as the evolution variable in the DGLAP approach. The most popular choice is the transfer momentum squared $Q^{2}$, or its logarithm $\xi=\ln \left(Q^{2} / Q_{0}^{2}\right)$. The double logarithm that explicitly takes into account the behavior of the effective coupling constant in the leading logarithm approximation proves very instrumental as well,

$$
t=\frac{2}{\beta} \ln \left[\frac{\ln \left(\frac{Q^{2}}{\Lambda^{2}}\right)}{\ln \left(\frac{Q_{0}^{2}}{\Lambda^{2}}\right)}\right],
$$

where $\beta=\left(11 N_{c}-2 n_{f}\right) / 3$ in $\mathrm{QCD}, Q_{0}$ is the some characteristic scale above which the perturbative theory is applicable, $n_{f}$ is the number of active flavors, $\Lambda$ is the QCD dimensional parameter, and $N_{c}=3$ is the color number. In Eq. (1), the one-loop running QCD coupling

$$
\alpha_{s}\left(Q^{2}\right)=\frac{4 \pi}{\beta \ln \left(Q^{2} / \Lambda^{2}\right)}
$$

was used.

The DGLAP evolution equations [1-4] assume the simplest form if we use the natural dimensionless evolution variable $t$; that is,

$$
\frac{d D_{h}^{j}(x, t)}{d t}=\sum_{j^{\prime}} \int_{x}^{1} \frac{d x^{\prime}}{x^{\prime}} D_{h}^{j^{\prime}}\left(x^{\prime}, t\right) P_{j^{\prime} \rightarrow j}\left(\frac{x}{x^{\prime}}\right) .
$$

These equations describe the evolution of single distributions $D_{h}^{j}(x, t)$ of bare quarks, antiquarks, and gluons $(j=q, \bar{q}, g)$ within a hadron $h$ in response to the change of the evolution variable $t$. The kernels, $P$, of these equations include a regularization at $x \rightarrow x^{\prime}$ and are known in their appropriate forms.

Equations (3) are explicitly solved by introducing the Mellin transforms

$$
M_{h}^{j}(n, t)=\int_{0}^{1} d x x^{n} D_{h}^{j}(x, t),
$$

which reduce those to a system of ordinary linear-differential equations at the first order,

$$
d M_{h}^{j}(n, t) / d t=\sum_{j^{\prime}} M_{h}^{j^{\prime}}(n, t) P_{j^{\prime} \rightarrow j}(n),
$$

where

$$
P_{j^{\prime} \rightarrow j}(n)=\int_{0}^{1} x^{n} P_{j^{\prime} \rightarrow j}(x) d x .
$$

To obtain the distributions in $x$ representation, the inverse Mellin transformation should be performed,

$$
x D_{h}^{j}(x, t)=\int \frac{d n}{2 \pi i} x^{-n} M_{h}^{j}(n, t),
$$

where the integration runs along the imaginary axis to the right from all $n$ singularities. It can be done in a general form numerically only. However, the asymptotic behavior can be estimated in some interesting and simple enough limits with the technique under consideration.

The solutions of the DGLAP equations with the given initial conditions $D_{h}^{j}(x, 0)$ at the reference scale $Q_{0}(t=0)$ can be expressed by the Green's functions $D_{i}^{j}(z, t)$ in the following way:

$$
D_{h}^{j}(x, t)=\sum_{i} \int_{x}^{1} \frac{d z}{z} D_{h}^{i}(z, 0) D_{i}^{j}\left(\frac{x}{z}, t\right) .
$$

These Green's functions (gluon distributions at the parton level) $D_{i}^{j}(z, t)$ are the solutions of Eqs. (3) at the parton level with the singular initial conditions $D_{i}^{j}(z, t=0)=$ $\delta(x-1) \delta_{i j}$ and in the double logarithm approximation (see, for instance, Refs. [3,9]) look like

$$
\begin{aligned}
x D_{g}^{g}(x, t) & =4 N_{c} t \exp [-a t] I_{1}(v) / v \\
& \simeq 4 N_{c} t v^{-3 / 2} \exp [v-a t] / \sqrt{2 \pi},
\end{aligned}
$$

where

$$
v=\sqrt{8 N_{c} t \ln (1 / x)}, \quad a=\frac{11}{6} N_{c}+\frac{1}{3} n_{f} / N_{c}^{2}
$$


and $I_{1}$ is the standard modified Bessel function. This result just illustrates the unitarity violation at very small $x$. In addition, one should also note that the mean number of partons of type $j$ in a parton of type $i$,

$$
\left\langle n^{j}\right\rangle_{i}=M_{i}^{j}(0, t)=[\exp P(0) t]_{i}^{j},
$$

cannot be correctly determined in the collinear approach because the kernels $P_{g \rightarrow g}(0)$ and $P_{q \rightarrow g}(0)$ are divergent and some improvements are necessary at very small $x$.

\section{COLLINEAR EVOLUTION WITH DISSIPATION}

The modification of collinear timelike evolution equations was discussed in Refs. $[19,20]$ to take into account the formation (so-called pionization) of soft quark-antiquark pairs at a hard quark (gluon) propagating. In analogy with the electron-photon showers, the energy outflow was phenomenologically simulated by the dissipative terms in the evolution equations with a rather interesting income. Such a modification for the spacelike evolution has, of course, another physical motivation in our case due to the parton diffusion in the rapidity space at large parton densities, and we suggest

$$
\begin{aligned}
\frac{\partial D_{i}^{j}(x, t)}{\partial t}= & \sum_{j^{\prime}} \int_{x}^{1} \frac{d x^{\prime}}{x^{\prime}} D_{i}^{j^{\prime}}\left(x^{\prime}, t\right) P_{j^{\prime} \rightarrow j}\left(\frac{x}{x^{\prime}}\right) \\
& +\gamma^{j} \frac{\partial D_{i}^{j}(x, t)}{\partial x}
\end{aligned}
$$

with $\gamma^{j}$ as some parameters characterizing the process of the energy outflow.

In the situation of small dissipation, $\gamma^{j} \ll 1$, the mean number of partons can be calculated [20] by using the Mellin technique. For credibility, we bring here the result for gluon multiplicity at the early evolution stage $(t \ll 1)$ only referring to the transparent, but laborious, calculations performed in Ref. [20],

$$
\begin{aligned}
\left\langle n^{g}\right\rangle_{g}= & I_{0}(V) e^{-a t} \\
& +\sqrt{\frac{2 N_{c} t}{\ln \left(1 / \gamma^{g}\right)}} \ln \sqrt{\frac{\ln \left(1 / \gamma^{g}\right)}{2 N_{c} t}} I_{1}(V) e^{-a t},
\end{aligned}
$$

where

$$
V=\sqrt{8 N_{c} t \ln \left(1 / \gamma^{g}\right)}
$$

and $I_{0}$ is another modified Bessel function. This result (13) reproduces exactly the mean number of gluons with the longitudinal momentum fractions larger than $x_{0}=\gamma^{g}$ as calculated in the DGLAP unmodified approach. The exercise above makes transparent the physical meaning of the dissipative term. It establishes the scale of energy drift because gluons (partons) with the longitudinal momentum fractions less than $\gamma^{g}$ are simply withdrawn from consideration. Moreover, the evolution is, in fact, frozen at the scale [19]

$$
Q_{f r}^{2}=\Lambda^{2}\left(Q^{2} / \Lambda^{2}\right)^{\gamma^{g}} .
$$

The origin of this freezing scale is similar to the saturation scale in the color glass condensate (CGC) approach $[13,15-18,21]$.

\section{GENERALIZING TO DOUBLE PARTON DISTRIBUTIONS}

The extension of basic equations to double parton distribution functions is straightforward:

$$
\begin{aligned}
& \frac{\partial D_{h}^{j_{1} j_{2}}\left(x_{1}, x_{2}, t\right)}{\partial t} \\
& =\sum_{j_{1}^{\prime}} \int_{x_{1}}^{1-x_{2}} \frac{d x_{1}^{\prime}}{x_{1}^{\prime}} D_{h}^{j_{1}^{\prime} j_{2}}\left(x_{1}^{\prime}, x_{2}, t\right) P_{j_{1}^{\prime} \rightarrow j_{1}}\left(\frac{x_{1}}{x_{1}^{\prime}}\right) \\
& \quad+\gamma^{j_{1}} \frac{\partial D_{h}^{j_{1} j_{2}}\left(x_{1}, x_{2}, t\right)}{\partial x_{1}} \\
& \quad+\sum_{j_{2}^{\prime}} \int_{x_{2}}^{1-x_{1}} \frac{d x_{2}^{\prime}}{x_{2}^{\prime}} D_{h}^{j_{1} j_{2}^{\prime}}\left(x_{1}, x_{2}^{\prime}, t\right) P_{j_{2}^{\prime} \rightarrow j_{2}}\left(\frac{x_{2}}{x_{2}^{\prime}}\right) \\
& \quad+\gamma^{j_{2}} \frac{\partial D_{h}^{j_{1} j_{2}}\left(x_{1}, x_{2}, t\right)}{\partial x_{2}} \\
& \quad+\sum_{j^{\prime}} D_{h}^{j^{\prime}}\left(x_{1}+x_{2}, t\right) \frac{1}{x_{1}+x_{2}} P_{j^{\prime} \rightarrow j_{1} j_{2}}\left(\frac{x_{1}}{x_{1}+x_{2}}\right) .
\end{aligned}
$$

Here, the splitting kernels,

$$
\frac{1}{x_{1}+x_{2}} P_{j^{\prime} \rightarrow j_{1} j_{2}}\left(\frac{x_{1}}{x_{1}+x_{2}}\right),
$$

which appear in the nonhomogeneous part of the equations, are the nonregularized one-loop well-known DGLAP kernels without the "+" prescription. The unmodified equations were derived first in Refs. [22,23] in framework of the DGLAP approach. The functions $D_{h}^{j_{1} j_{2}}\left(x_{1}, x_{2}, t\right)$ in question have a specific interpretation in the leading logarithm approximation of perturbative QCD. They are the inclusive probabilities that allow one to find two bare partons of types $j_{1}$ and $j_{2}$ with the given longitudinal momentum fractions $x_{1}$ and $x_{2}$ in a hadron $h$.

The dissipative terms provide the energy outflow and establish the scale of energy drift as well. Gluons (partons) with the longitudinal momentum fractions less than $\gamma^{j}$ are simply removed again from consideration for each of two parton cascade branches practically independently. In the small- $x$ region, we can restrict ourselves to homogeneous 
evolution equations because the solutions of nonhomogeneous unmodified equation are substantial at not parametrically small longitudinal momentum fractions only [24]. Moreover, the homogeneous evolution equations (independent evolution of two branches) admit the factorization of double parton distribution functions,

$$
D_{h}^{j_{1} j_{2}}\left(x_{1}, x_{2}, t\right) \simeq D_{h}^{j_{1}}\left(x_{1}, t\right) D_{h}^{j_{2}}\left(x_{2}, t\right),
$$

as a good approximate solution, if such a factorization was assumed at the reference scale $Q_{0}(t=0)$.

Further, we hold the leading exponential terms only if those have the same structure [25] both at the parton level and the hadron level under smooth enough initial conditions at the reference scale. Indeed, Eq. (8) in the double logarithm approximation reads

$$
\begin{aligned}
x D_{h}^{g}(x ; t) \simeq & \left.\int_{0}^{Y} d y\left[z D_{h}^{g}(z, 0)\right]\right|_{1 / z=\exp y} \\
& \times \exp \left[\sqrt{8 N_{c}} \sqrt{t(Y-y)}\right] \\
\sim & \exp \left[\sqrt{8 N_{c}} \sqrt{t Y}\right],
\end{aligned}
$$

with $Y=\ln (1 / x)$. The $y$ integration is not a saddle-point type, and therefore one of the edges, just $y \rightarrow 0(z \rightarrow 1)$, dominates, provided that the initial gluon distribution does not increase too much with $z$ decreasing. Actually, one needs $z D_{h}^{g}(z, 0) \sim(1 / z)^{a}$ at $z \rightarrow 0$ with $a<A$, where $A=\sqrt{2 N_{c} t / Y}>0$. Let us notice that the parametrization of the initial gluon distributions, usually used, satisfies this condition (e.g., the parametrization of CTEQ Collaboration from Ref. [26]). Thus, as a result, we have for the double gluon distributions [25] in this approximation

$$
\begin{aligned}
& x_{1} x_{2} D_{h}^{g g}\left(x_{1}, x_{2}, t\right) \\
& \quad \sim \exp \left[\sqrt{8 N_{c}}\left(\sqrt{t \ln \left(1 / x_{1}\right)}+\sqrt{t \ln \left(1 / x_{2}\right)}\right)\right],
\end{aligned}
$$

with the infinite mean number of such gluons. If the two branches evolve independently, then introducing the dissipative terms slows down the rate of gluon density increase, and one gets the finite mean gluon numbers as

$\left\langle n^{g g}\right\rangle_{h} \sim \exp \left[\sqrt{8 N_{c}}\left(\sqrt{t \ln \left(1 / \gamma^{g}\right)}+\sqrt{t \ln \left(1 / \gamma^{g}\right)}\right)\right]$

since the gluons with the longitudinal momentum fractions less than $\gamma^{g}$ are simply excluded.

\section{CONCLUSIONS}

Clearly, the dissipative parameters above cannot be determined within the DGLAP approach. They are treated as the phenomenological parameters in numerical simulations and should be estimated in the other models for further applications. The phenomena of saturation and slowing down an increase of gluon density take place also in the CGC scenario [13,15-18]. However, the saturation scale is energy dependent in that approach, and, nevertheless, it turns out quite predictive. For example, in the Golec-Biernat-Wusthoff model [27,28], it is parametrized by three parameters,

$$
Q_{s}^{2}=Q_{0}^{2}\left(x_{0} / x\right)^{\lambda},
$$

with $Q_{0}=1 \mathrm{GeV}, x_{0} \simeq 0.0001$, and $\lambda \simeq 0.3$, which have been used to accurately describe the HERA data [29]. The value of characteristic energy (longitudinal momentum fraction) $x_{0}$ in Eq. (22) allows us to estimate the dissipative parameter $\gamma^{g}$ that has a physical meaning similar to $x_{0}$. In fact, it justifies the assumption of small dissipation used in the previous sections to obtain the crucial estimates (13) and (21), which are pretty encouraging to investigate the properties of modified collinear equations further as a new alternative insight into the saturation physics extending the initial limits of the linear approach.

In summary, the modified collinear evolution equations are suggested to extract information on the properties of a hot and dense QCD medium created in the experiments on heavy ion collisions searching the quark-gluon plasma, a thermalized phase, that may exist in very specific regimes for very short periods of time. Comprehensive phenomenological analysis of proton-proton collisions based on the QCD factorization, as a key instrument, made it possible to extract the universal distribution functions validating such an approximation and open up (quite often) transparent ways for introducing the efficient corrections. Truly, these corrections at leading power of the large momentum transfer are fairly general and easily traceable, but the corrections within the factorized forms turn out very complicated and too sensitive to the process details, as it was shown again many years ago, because of the QCD multiple scatterings in hadronic collisions differ from heavy ion ones significantly [30]. The model presented in this paper shows the possibility of perturbatively forming a dynamical regime in a particular kinematical configuration that could not be foreseen according to the theoretical dogmas. As argued, it concerns a regime of high parton densities and dynamical interactions described definitely by nonlinear equations. The evolution of hadron scattering amplitudes, at least, in the framework of the color dipole picture in such a regime is quite similar [31] to the time evolution of a classical particles system undergoing reaction-diffusion processes. Amazingly, introducing the dissipative terms results in an origin of the natural saturation scale on which the evolution is frozen and the gluons with longitudinal momentum less than $\gamma^{g}$ are simply excluded. In the phenomenological applications, the direct numerical solutions of suggested modified equations may occur simpler than the BFKL treatment of the very small- $x$ region. 


\section{ACKNOWLEDGMENTS}

Inspiring discussions (many years ago) with I. M. Dremin, A. Kovner, A. V. Leonidov, and L. McLerran are gratefully acknowledged. The work of G. M. is supported by the Goal-Oriented Program of Cooperation between the European Organization for Nuclear Research (CERN) and National Academy of Science of Ukraine "Nuclear Matter under Extreme Conditions" (agreement CC/1-2019, Grant No. 0118U005343).
[1] V. N. Gribov and L. N. Lipatov, Yad. Fiz. 15, 781 (1972) [Sov. J. Nucl. Phys. 15, 438 (1972)]; Yad. Fiz. 15, 1218 (1972) [Sov. J. Nucl. Phys. 15, 675 (1972)].

[2] L. N. Lipatov, Yad. Fiz. 20, 181 (1974) [Sov. J. Nucl. Phys. 20, 94 (1974)].

[3] Y. L. Dokshitzer, Zh. Eksp. Teor. Fiz. 73, 1216 (1977) [Sov. Phys. JETP 46, 641 (1977)].

[4] G. Altarelli and G. Parisi, Nucl. Phys. B126, 298 (1977).

[5] E. A. Kuraev, L. N. Lipatov, and V. S. Fadin, Zh. Eksp. Teor. Fiz. 71, 840 (1976) [Sov. Phys. JETP 44, 443 (1976)].

[6] E. A. Kuraev, L. N. Lipatov, and V. S. Fadin, Zh. Eksp. Teor. Fiz. 72, 377 (1977) [Sov. Phys. JETP 45, 199 (1977)].

[7] I. I. Balitsky and L. N. Lipatov, Yad. Fiz. 28, 1597 (1978) [Sov. J. Nucl. Phys. 28, 822 (1978)].

[8] V. Khachatryan and M. Praszalowicz, arXiv:1907.03815.

[9] L. V. Gribov, E. M. Levin, and M. G. Ryskin, Nucl. Phys. 188, 555 (1981); Phys. Rep. 100, 1 (1983).

[10] A. H. Mueller and J. W. Qui, Nucl. Phys. 268, 427 (1986).

[11] L. N. Lipatov, Phys. Rep. 286, 131 (1997).

[12] A. H. Mueller, Nucl. Phys. B437, 107 (1995); in Particle Production Spanning MeV and TeV Energies, edited by W. Kittel, P. J. Mulders, and O. Scholten, NATO Science Series Vol. 554 (Springer, Dordrecht, 2000), pp. 71-99.

[13] L. McLerran and R. Venugopalan, Phys. Rev. D 49, 2233 (1994); 49, 3352 (1994); 50, 2225 (1994).

[14] Y. V. Kovchegov, Phys. Rev. D 60, 034008 (1999); Nucl. Phys. A854, 3 (2011).

[15] E. Iancu and R. Venugopalan, in Quark Gluon Plasma, edited by R. G. Hwa et al. (World Scientific, Singapore, 2003), p. 249.
[16] F. Gelis, Int. J. Mod. Phys. A 28, 1330001 (2013).

[17] A. Dumitru, Nucl. Phys. A904-A905, 51 (2013).

[18] J.-P. Blaizot, Rep. Prog. Phys. 80, 032301 (2017).

[19] I. M. Dremin, Pis'ma Zh. Eksp. Teor. Fiz. 31, 201 (1980).

[20] I. M. Dremin and A. V. Leonidov, Yad Fiz. 35, 430 (1982) [Sov. J. Nucl. Phys. 35, 247 (1982)].

[21] E. Iancu, A. Leonidov, and L. McLerran, Lectures at the NATO Advanced Study Institute QCD Perspectives on Hot and Dense Matter in Cargese, Corsica, France (Kluwer Academic, Dordrecht, Netherlands, 2001).

[22] R. Kirschner, Phys. Lett. B 84, 266 (1979).

[23] V.P. Shelest, A. M. Snigirev, and G. M. Zinovjev, Phys. Lett. B 113, 325 (1982); Teor. Mat. Fiz. 51, 317 (1982) [Theor. Math. Phys. 51, 523 (1982)].

[24] A. M. Snigirev, N. A. Snigireva, and G. M. Zinovjev, Phys. Rev. D 90, 014015 (2014).

[25] M. G. Ryskin and A. M. Snigirev, Phys. Rev. D 86, 014018 (2012).

[26] J. Pumplin, D. R. Stump, J. Huston, H.-L. Lai, P. Nadolsky, and W.-K. Tung, J. High Energy Phys. 07 (2002) 012.

[27] K. Golec-Biernat and M. Wusthoff, Phys. Rev. D 59, 014017 (1998).

[28] K. Golec-Biernat and S. Sapeta, J. High Energy Phys. 03 (2018) 102.

[29] H. Abramowicz et al. (ZEUS and H1 Collaboration), Eur. Phys. J. C 75, 580 (2015).

[30] B. G. Zakharov, JETP Lett. 63, 952 (1996).

[31] R. Peschanski, arXiv:hep-ph/0312043. 